\title{
Journal-club pour le praticien \\ Journal-Klub für den Praktiker
}

Die Lokalisierung von Hämangiomen im Gesicht: mit System, nicht zufalls-

\section{bedingt}

[Arch Dermatol 2003;139:869]

Die Verteilung von Hämangiomen im Gesicht gibt im Kreise der Familie Betroffener seit jeher Anlass zu wilden Spekulationen. Das Forschungsteam von Martin Mihm, das vor einiger Zeit die Vermutung geäussert hatte, Hämangiome seien Metastasen der Plazenta (siehe «Dermatologica Helvetica» $6 / 2001$ ), veröffentlicht nun eine topographische Untersuchung zum Thema. Man unterscheidet zwei Arten von Hämangiomen im Gesicht: das häufigere fokale Hämangiom, das ein tumorartiges Aussehen besitzt, und das plaqueförmige
Hämangiom. Fokale Hämangiome treten bevorzugt an embryologischen Fusionsstellen auf, während plaqueförmige Hämangiome eine segmentartige Anordnung erkennen lassen. Diese Fortschritte bezüglich der biologischen Grundlagen von Hämangiomen künden bessere Behandlungsmöglichkeiten an.

\section{Algen d a}

\section{Möchten Sie hier einen Kongress anzeigen?}

Bitte schicken Sie vollständige Angaben (genaues Datum, Ort, Titel der Veranstaltung, Kontaktadresse einschliesslich E-Mail) für diese Rubrik an: dermatologica.helvetica@hcuge.ch. Danke.

\section{Voudriez-vous annoncer un congrès?}

Veuillez envoyer, s.v.p., les spécifications complètes (date exacte, lieu, thème du congrès, adresse de contact avec E-mail) pour cette rubrique à: dermatologica.helvetica@hcuge.ch.

\section{Im Ausland/A I'étranger}

\section{Week 18}

April 28 to May 1, 2004

65th Annual Meeting of the Society for Investigative Dermatology, Providence, RI, USA

$\begin{array}{ll}\text { Meeting } & \text { Kate Rader } \\ \text { manager: } & \text { The Society of Investigative Dermatology } \\ & \text { 820 West Superior Ave., 7th Floor } \\ & \text { Cleveland, OH 44113 (USA) } \\ & \text { Tel. +1 216 579 9344, Fax +1 216 5799333 } \\ & \text { E-Mail krader@sidnet.org } \\ & \text { www.sidnet.org }\end{array}$

\section{Week 21}

May 19-22, 2004

9th International Congress of Dermatology, Beijing, China

Contact: International Congress Secretariat Tel. +8610 65249989, ext. 1606 Fax +861065123754

E-Mail ICD2004@chinamed.com.cn
Week 25

June 17-19, 2004

4th Intercontinental Meeting of Hair Research Societies, Berlin, Germany

Information:

\section{Der-Congress}

Congressorganisation

Bundesallee 56

DE-10715 Berlin (Germany)

Tel. +49 3085790 30, Fax +49 3084790326

E-Mailder@der-congress.de

Week 28/29

July 8-13, 2004

2nd World Union of Wound Healing Societies Meeting, Paris, France

Registration online: www.mfgroupe.com

Deadline for abstract submission before February 1, 2004

Information: Secretariat

8 , rue Tronchet

FR-75008 Paris (France)

Tel. +33140071121, Fax +33140071094

E-Mail congres@mfgroupe.com

\section{Week 47}

November 17-21, 2004

13th Congress of the European Academy of Dermatology and Venereology, Florence, Italy

'The Renaissance of Dermatology'

Information: $\quad$ Fax +390572 912280

E-Mail president@eadv2004.org/

info@eadv2004.org

www.EADV2004.org 